\title{
CEPILLADO DENTAL Y NIVELES DE FLÚOR EN PASTAS DENTALES USADAS POR NIÑOS PERUANOS MENORES DE 12 AÑOS
}

\author{
Akram Hernández-Vásquez ${ }^{1, a}$, Diego Azañedo $2, b$
}

\begin{abstract}
RESUMEN
Análisis secundario de los datos de 41330 niños de uno a 11 años de la Encuesta Demográfica y de Salud Familiar (ENDES) 2018. Se reportó la frecuencia de cepillado y el uso de pasta dental según el nivel de flúor (no cepillado, adecuado, inadecuado, no mostró/no usa pasta, e ilegible), según variables socioeconómicas y geográficas. Un 7,8\% de los niños evaluados no se cepillaba los dientes, se evidenció diferencias porcentuales significativas $(p<0,001)$ en el uso de pasta dental con concentración inadecuada de flúor entre las categorías extremas de las variables edad $(12,3 \%)$, uso de servicios odontológicos $(7,7 \%)$, nivel educativo del responsable $(20,4 \%)$, índice de riqueza $(17,8 \%)$, dominio geográfico $(11,3 \%)$ y área de residencia $(9,1 \%)$. Un porcentaje considerable de niños peruanos no se cepilla los dientes; además, un gran número de niños que se cepillan emplean pastas dentales con contenidos de flúor sin efecto preventivo.
\end{abstract}

Palabras Clave: Cepillado Dental; Pastas de Dientes; Niño; Preescolar; Perú (fuente: DeCS BIREME).

\section{TOOTH BRUSHING AND FLUORIDE LEVELS IN TOOTHPASTE USED BY PERUVIAN CHILDREN UNDER 12 YEARS OLD}

\begin{abstract}
Secondary analysis of data on 41,330 children aged one to 11 from the Demographic and Family Health Survey (ENDES) carried out in 2018. The frequency of brushing and the use of toothpaste were reported according to the level of fluoride (no brushing, adequate, inadequate, did not show/did not use toothpaste, and illegible), according to socioeconomic and geographical variables. The evaluation showed that $7.8 \%$ of children did not brush their teeth; there were significant percentage differences $(p<0.001)$ in the use of toothpaste with inadequate fluoride concentration between the extreme categories of the variables: age $(12.3 \%)$, use of dental services $(7.7 \%)$, educational level of the person responsible $(20.4 \%)$, wealth index (17.8\%), geographical domain (11.3\%), and area of residence $(9.1 \%)$. A considerable percentage of Peruvian children do not brush their teeth; in addition, a large number of children who brush use toothpastes with a fluoride content that has no preventive effect.
\end{abstract}

Keywords: Toothbrushing; Toothpastes; Child; Child, Preschool; Peru (source: MeSH NLM).

\section{INTRODUCCIÓN}

Según la Organización Mundial de la Salud, las enfermedades orales son el grupo más común de enfermedades no transmisibles ${ }^{(1)}$. A nivel mundial, casi cuatro mil millones de personas padecen enfermedades de la cavidad oral, la caries dental es la más prevalente y afecta a 500 millones de niños ${ }^{(2)}$. En Perú, la prevalencia de caries oscila entre $80 \%$ y $90 \%$ en niños entre tres y 15 años ${ }^{(3,4)}$, por lo que es necesario fortalecer y promover las diversas medidas preventivo-promocionales que han mostrado efectividad ante esta enfermedad ${ }^{(5)}$.

La medida más efectiva para la prevención de la caries es la exposición a flúor ${ }^{(5)}$, que inhibe la producción bacteriana de ácidos y frena la desmineralización del esmalte dental ${ }^{(5)}$. Por ello, la práctica de cepillado con pasta dental fluorada, es uno de los métodos más costo-efectivos y recomendados ${ }^{(5-7)}$. Las pastas dentales fluoradas que son eficaces en reducir el riesgo de caries

\footnotetext{
Universidad San Ignacio de Loyola, Vicerrectorado de Investigación, Centro de Excelencia en Investigaciones Económicas y Sociales en Salud. Lima, Perú

Universidad Católica Los Ángeles de Chimbote, Instituto de Investigación. Chimbote, Perú

Médico cirujano, magíster en Gestión y Políticas Públicas; ${ }^{\mathrm{b}}$ cirujano dentista

Recibido: 21/10/2019 Aprobado: 06/11/2019 En línea: 03/12/2019

Los códigos incluidos en el artículo están disponibles en el repositorio GitHub: https://github.com/ahernandezv/Fluor_ENDES_Peru y OSF: https://osf.io/tdk83/
}

Citar como: Hernández-Vásquez A, Azañedo D. Cepillado dental y niveles de flúor en pastas dentales usadas por niños peruanos menores de 12 años. Rev Peru Med Exp Salud Publica. 2019;36(4):646-52. doi: 10.17843/rpmesp.2019.364.4900. 
contienen más de 1000 partes por millón (ppm) de flúor, y su uso debe ser en cantidades adecuadas según la edad ${ }^{(6,8)}$

En 2001, el Perú aprobó la «Norma Técnica Sanitaria para la Adición de Fluoruros en Cremas Dentales, Enjuagatorios y otros productos utilizados en la Higiene Bucal», que establece que las pastas dentales para menores de seis años deben contener entre 250 a 550 ppm de flúor, y concentraciones mayores a 1100 ppm de flúor para adultos y niños mayores a seis años ${ }^{(9)}$. Contrario a esta norma, en el 2017, la «Guía de Práctica Clínica para la Prevención, Diagnóstico y Tratamiento de la Caries Dental en Niñas y Niños» del Ministerio de Salud (MINSA), recomienda el uso de pastas dentales con concentraciones de 1000 a 1500 ppm de flúor a partir de la primera erupción dental ${ }^{(10)}$. No obstante, y a pesar de las evidencias y recomendaciones ${ }^{(6,8,10)}$, algunos estudios reportan que casi la mitad de las pastas dentales vendidas en Lima tenían cantidades de flúor debajo de lo recomendado ${ }^{(11,12)}$.

Si bien, a nivel mundial el cepillado con dentífricos fluorados se ha incrementado en las últimas décadas, la población de menores ingresos, residentes de áreas rurales, bajo nivel educativo y con menor acceso a servicios de salud oral, usan dentífrico con concentraciones inadecuadas de flúor (menos de 1000 ppm) ${ }^{(5,13,14)}$. Esto se puede atribuir al desconocimiento de la población, transferencia de información desactualizada de parte del personal de salud e incapacidad para adquirir dentífricos con cantidades de flúor adecuadas, poniendo a la población en un riesgo mayor de padecer caries ${ }^{(7)}$. Asimismo, el $15 \%$ de niños peruanos menores de 12 años no se cepillan los dientes diariamente ${ }^{(15)}$.

En Perú, la Encuesta Demográfica y de Salud Familiar (ENDES) 2018, recoge información sobre la práctica de cepillado y nivel de flúor en la pasta dental; sin embargo, dicha información no está disponible en su informe anual (https://bit.ly/30OyM2S). Esta información sería importante para poder focalizar estrategias de promoción a nivel de la población, odontólogos, fabricantes y reguladores. Por ello, el presente estudio tuvo por objetivo analizar la distribución del cepillado y el nivel de flúor en la pasta dental según variables socioeconómicas y geográficas en niños peruanos menores de 12 años, utilizando la ENDES 2018.

\section{EL ESTUDIO}

La ENDES 2018 es una encuesta de base poblacional con un tamaño muestral de 36760 viviendas (35 502 viviendas entrevistadas) que provee información sobre la dinámica demográfica y el estado de salud de la población peruana. La población objetivo estuvo constituida por: a) Los hogares particulares y sus miembros, personas que son residentes habituales y aquellas que no siendo residentes pernoctaron

\section{MENSAJES CLAVE}

Motivación para realizar el estudio. La guía vigente de prevención en salud bucal en Perú, establece el uso de pastas dentales con concentración de flúor mayor a 1000 ppm en niños. Actualmente no se dispone de información representativa nacional respecto a la práctica de cepillado y nivel de flúor en pastas dentales utilizadas por niños menores de 12 años.

Principales hallazgos. Un 7,8\% de niños peruanos no se cepilla los dientes y muchos de los que se cepillan emplean pastas dentales con contenido inadecuado.

Implicancias. Se requiere fortalecer la promoción de cepillado en niños y la regulación en el nivel de flúor en las pastas dentales que se expenden en Perú.

en la vivienda la noche anterior al día de la entrevista; b) Una persona de 15 años a más de edad por cada hogar particular; c) Todas las niñas y niños menores de 12 años. Más detalles sobre el diseño y muestreo de la ENDES 2018 está disponible en la ficha técnica de la encuesta (https://bit. ly/300yM2S).

Un total de 45487 menores de 12 años participaron en la ENDES 2018. Nuestro análisis incluyó una submuestra de menores entre uno y 11 años $(n=41330)$ con información completa en todas las variables de interés (Figura 1). La selección del grupo etario evaluado se basó en la recomendación de inicio del cepillado con pasta dental fluorada desde la erupción del primer diente, según la guía de práctica clínica vigente y de aplicación en todo el ámbito nacional ${ }^{(10)}$. Se excluyeron a los menores de un año considerando que la edad de la primera erupción dentaria es variable (seis a diez meses desde el nacimiento).

Se consideró como variable de interés a la práctica de cepillado con el uso de pasta dental según el nivel de flúor definiendo las siguientes categorías: no cepillado, adecuado nivel de flúor (1000 ppm o más), inadecuado nivel de flúor (menos de 1000 ppm), no mostró pasta/no usa pasta, y nivel de flúor ilegible (incluye: no tiene información, número borroso, valor diferente a ppm, y otro). El nivel de flúor fue determinado por las encuestadoras mediante observación directa del tubo de pasta dental (pregunta textual: Por favor, ¿Podría mostrarme el cepillo dental y la crema dental con que (NOMBRE) se cepilla los dientes?) y el cepillado dental fue reportado por el responsable del cuidado del menor ante la pregunta « $i(N O M B R E)$ se lava los dientes con cepillo dental?». Las variables de estratificación fueron el sexo del menor, edad del menor categorizado en 1-5 años y 6-11 años, uso de servicios odontológicos (menos de seis meses, y más de seis meses), tenencia de seguro de salud (sí/no), nivel educativo del responsable del menor (sin nivel educativo, primaria, secundaria y superior), índice de riqueza (quintil inferior, segundo quintil, quintil intermedio, 


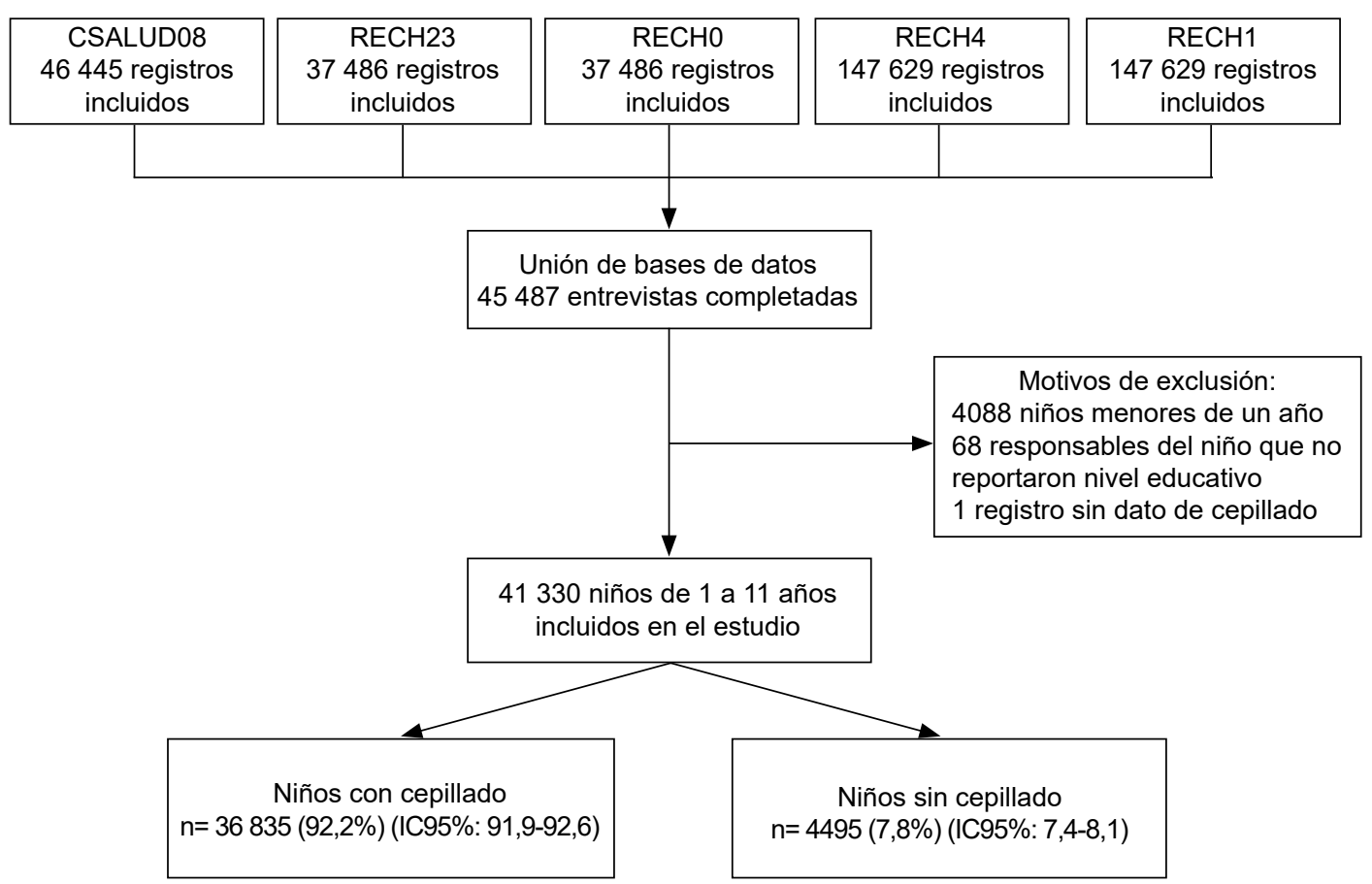

Figura 1. Flujograma de la selección de participantes incluidos en el estudio

cuarto quintil, quintil superior), dominio geográfico (Lima Metropolitana, resto de Costa, Sierra, Selva), y área de residencia (urbana/rural).

Las bases de datos fueron descargadas del sitio web http:// inei.inei.gob.pe/microdatos/, importadas y procesadas con R (versión 3.6.1, R Foundation for Statistical Computing, Vienna, Austria) y RStudio (versión 1.2.1335, RStudio, Inc., Boston, MA). Todas las estimaciones incluyeron las características del diseño muestral complejo y los factores de ponderación de la ENDES 2018 para mostrar proporciones ponderadas con intervalos de confianza al 95\% para cada una de las categorías incluidas. Para el análisis de diferencias entre categorías de las variables se empleó una combinación lineal de los parámetros mediante una prueba $t$, fijando un valor de $p<0,05$ como estadísticamente significativo.

El script con la construcción de las variables, librerías y códigos empleados para el análisis está disponible en GitHub https://github.com/ahernandezv/Fluor_ENDES_ Peru y OSF https://osf.io/tdk83/, lo cual permite la reproducibilidad de los resultados.

La realización del estudio no requirió la aprobación de un comité de ética por tratarse de un análisis de datos secundarios que son de dominio público y de libre acceso (http://iinei.inei.gob.pe/microdatos/) que no permite identificar a los participantes.

\section{RESULTADOS}

Del total de niños incluidos en el estudio (41 330), el $50,8 \%$ fueron varones, $55,3 \%$ pertenecieron al grupo etario de 6 a 11 años, $49 \%$ eran pobres o muy pobres, $15,3 \%$ no estaban afiliados a un seguro de salud y $74,4 \%$ vivían en el área urbana (Tabla 1 ).

Un 7,8\% del total de niños incluidos en el estudio no se cepillaba los dientes, además, se observaron los más altos porcentajes de no cepillado dental en los niños de uno a cinco años (15,8\%), aquellos atendidos en un servicio odontológico en un periodo mayor a seis meses $(9,9 \%)$, niños menores con responsables sin nivel educativo $(12,7 \%)$, aquellos pertenecientes al quintil inferior de riqueza $(12,2 \%)$, residentes de la Sierra $(9,7 \%)$ y de las zonas rurales $(11,9 \%)$ (Tabla 2$)$.

Se evidenciaron mayores porcentajes de uso de pasta dental con concentración inadecuada de flúor en niños de uno a cinco años (27,0\%), aquellos que utilizaron el servicio odontológico durante los seis meses previos a la encuesta $(25,3 \%)$, niños de padres con nivel educativo superior $(28,8 \%)$, menores pertenecientes al quintil superior de riqueza $(29,5 \%)$, residentes de Lima Metropolitana $(23,7 \%)$, y residentes de zonas urbanas $(22,4 \%)$ (Tabla 2$)$. Asimismo, se evidenció diferencias porcentuales estadísticamente significativas $(p<0,001)$ 
Tabla 1. Descripción de la muestra, Encuesta Demográfica $y$ de Salud Familiar (ENDES) $2018(n=41330)$

\begin{tabular}{|c|c|c|}
\hline Característica & $\mathbf{n}$ & $\%(\mathrm{IC} 95 \%)$ * \\
\hline \multicolumn{3}{|l|}{ Sexo } \\
\hline Hombre & 20966 & $50,8(50,1-51,5)$ \\
\hline Mujer & 20364 & $49,2(48,5-49,9)$ \\
\hline \multicolumn{3}{|l|}{ Edad (años) } \\
\hline $1-5$ & 23296 & $44,7(44,1-45,2)$ \\
\hline $6-11$ & 18034 & $55,3(54,8-55,9)$ \\
\hline \multicolumn{3}{|c|}{$\begin{array}{l}\text { Uso de servicio odontológico } \\
\text { (meses) }\end{array}$} \\
\hline$\leq 6$ & 13196 & $31,9(31,1-32,7)$ \\
\hline$>6$ & 28134 & $68,1(67,3-68,9)$ \\
\hline \multicolumn{3}{|c|}{ Tenencia de seguro de salud } \\
\hline Sí & 36041 & $84,7(84,0-85,4)$ \\
\hline No & 5289 & $15,3(14,6-16,0)$ \\
\hline \multicolumn{3}{|l|}{$\begin{array}{l}\text { Nivel educativo del } \\
\text { responsable }\end{array}$} \\
\hline Sin nivel educativo & 1273 & $2,8(2,5-3,1)$ \\
\hline Primaria & 11048 & $24,1(23,3-25,0)$ \\
\hline Secundaria & 17722 & $42,7(41,7-43,7)$ \\
\hline Superior & 11287 & $30,4(29,5-31,3)$ \\
\hline \multicolumn{3}{|c|}{ Índice de riqueza del hogar } \\
\hline Muy pobre & 13799 & $25,5(24,6-26,4)$ \\
\hline Pobre & 10656 & $23,3(22,4-24,2)$ \\
\hline Medio & 7555 & $20,0(19,2-20,8)$ \\
\hline Rico & 5450 & $16,9(16,1-17,7)$ \\
\hline Muy rico & 3870 & $14,3(13,5-15,1)$ \\
\hline \multicolumn{3}{|c|}{ Dominio geográfico de residencia } \\
\hline Lima Metropolitana & 4682 & $29,9(28,9-30,8)$ \\
\hline Resto de la Costa & 11596 & $25,7(24,7-26,7)$ \\
\hline Sierra & 14343 & $27,2(26,1-28,4)$ \\
\hline Selva & 10709 & $17,2(16,3-18,1)$ \\
\hline \multicolumn{3}{|l|}{ Área de residencia } \\
\hline Urbana & 27069 & $74,4(73,7-75,1)$ \\
\hline Rural & 14261 & $25,6(24,9-26,3)$ \\
\hline
\end{tabular}

* Las estimaciones incluyeron el factor de ponderación y especificaciones muestrales de la ENDES 2018

IC95\%: intervalo de confianza al 95\%

en el uso de pasta dental con concentración inadecuada de flúor entre las categorías extremas de las variables edad (12,3\%), uso de servicios odontológicos $(7,7 \%)$, nivel educativo del responsable $(20,4 \%)$, índice de riqueza en el hogar $(17,8 \%)$, dominio geográfico de residencia $(11,3 \%)$ y área de residencia $(9,1 \%)$ (Tabla 2 ).

\section{DISCUSIÓN}

Se encontró que un $7,8 \%$ de los niños peruanos no se cepilla los dientes. Asimismo, se evidenció gradientes respecto al uso de pasta dental con concentración inadecuada de flúor entre las categorías extremas de las variables edad, uso de servicios odontológicos, nivel educativo del responsable, índice de riqueza en el hogar, dominio geográfico de residencia y área de residencia. Estos resultados dan cuenta del bajo cumplimiento de las recomendaciones internacionales y del MINSA sobre el contenido adecuado de flúor en las pastas dentales para prevenir la caries, incluso desde el momento de la primera erupción dental ${ }^{(6-8,10)}$.

Los niños de uno a cinco años tuvieron una mayor frecuencia de no cepillado que los de seis a 12 años, tendencia reportada anteriormente por otros estudios ${ }^{(16,17)}$, esto podría ser atribuido a que los niños en esa edad requieren de ayuda para cepillarse y sus padres pueden no estar conscientes de ello y restarle importancia al cepillado en la dentición decidua (18). El porcentaje de no cepillado fue mayor en aquellos no atendidos en un servicio odontológico dentro de los seis meses previos, similar a lo reportado en un estudio de niños mexicanos de seis a 12 años, que reportó una mayor probabilidad de cepillado frecuente en aquellos que recibieron atención odontológica en los 12 meses previos al estudio ${ }^{(19)}$. Estos hallazgos mostrarían que el contacto periódico con el odontólogo sirve para reforzar el hábito del cepillado en niños.

Por otro lado, el porcentaje de no cepillado fue mayor en niños cuyos responsables tuvieron menor nivel educativo, siendo esto consistente con lo reportado en otros estudios donde un menor nivel educativo del padre jefe de familia está asociado con un inicio tardío del cepillado dental, y la frecuencia y duración del cepillado de sus hijos (20). Asimismo, un menor nivel educativo familiar está relacionado a un menor índice de riqueza del hogar, característica que en nuestro país se concentra en la Sierra y en zonas rurales (https://bit.ly/2rsluaO). Esto es consistente con nuestros hallazgos, pues el no cepillado fue mayor en el quintil inferior de riqueza, y en aquellos niños residentes de la Sierra, y de zonas rurales.

El uso de pastas con concentración de flúor inadecuada fue mayor en $12,3 \%$ en los niños de menor edad, posiblemente debido a la vigencia de la norma técnica del 2001, que establece el uso de pastas con 250-550 ppm de flúor en niños menores de seis años ${ }^{(9)}$, pudiendo haber odontólogos que aún recomiendan este tipo de pastas a sus pacientes pediátricos. Además, puede que los padres adquieran pastas con concentraciones inadecuadas de flúor ya que generalmente estas consignan la etiqueta de «para niños» o «kids» y recomiendan su uso en menores de seis años ${ }^{(11,12)}$. Asimismo, los niños que recibieron atención odontológica en un periodo menor a seis meses, presentaron mayor frecuencia de uso inadecuado de flúor, lo cual puede estar relacionado a la información proporcionada por los odontólogos que puede estar basada en la norma vigente.

Las mayores proporciones de uso de concentraciones inadecuadas de flúor se encontraron en aquellos niños 
Tabla 2. Cepillado y nivel de flúor en pasta dental, Encuesta Demográfica y de Salud Familiar (ENDES) 2018

\begin{tabular}{|c|c|c|c|c|c|c|c|}
\hline \multirow[b]{2}{*}{ Característica } & \multicolumn{5}{|c|}{ Cepillado y nivel de flúor en pasta dental, \% (IC95\%) } & \multirow{2}{*}{$\begin{array}{c}\text { Diferencia A } \\
\text { Diferencia } \\
\text { (Valor de } p)^{*}\end{array}$} & \multirow{2}{*}{$\begin{array}{c}\text { Diferencia B } \\
\text { Diferencia } \\
\text { (Valor de } \mathrm{p})^{*}\end{array}$} \\
\hline & No cepillado & Adecuada & Inadecuada & $\begin{array}{c}\text { No mostró/no } \\
\text { usa }\end{array}$ & llegible & & \\
\hline Total & $7,8(7,4-8,1)$ & $44,2(43,2-45,1)$ & $20,1(19,3-20,8)$ & $25,0(24,2-25,9)$ & $2,9(2,7-3,2)$ & - & \\
\hline \multicolumn{8}{|l|}{ Sexo del menor } \\
\hline Hombre & $7,8(7,4-8,3)$ & $44,9(43,7-46,0)$ & $19,6(18,7-20,5)$ & $24,6(23,6-25,5)$ & $3,1(2,7-3,4)$ & $0,1(0,709)$ & $1,0(0,800)$ \\
\hline Mujer & $7,7(7,3-8,2)$ & $43,4(42,3-44,5)$ & $20,6(19,6-21,5)$ & $25,5(24,5-26.6)$ & $2,8(2,4-3,1)$ & & \\
\hline \multicolumn{8}{|l|}{ Edad del menor (años) } \\
\hline $1-5$ & $15,8(15,2-16,5)$ & $28,5(27,5-29,4)$ & $26,9(26,0-27,8)$ & $23,9(23,1-24,8)$ & $4,9(4,5-5,4)$ & $14,5(<0,001)$ & $12,3(<0,001)$ \\
\hline 6-11 & $1,3(1,0-1,6)$ & $56,8(55,6-58,1)$ & $14,6(13,7-15,5)$ & $26,0(24,9-27,1)$ & $1,3(1,1-1,6)$ & & \\
\hline \multicolumn{8}{|l|}{$\begin{array}{l}\text { Uso de servicio } \\
\text { odontológico (meses) }\end{array}$} \\
\hline$\leq 6$ & $3,1(2,8-3,5)$ & $44,4(43,0-45,9)$ & $25,3(24,0-26,5)$ & $24,1(22,7-25,3)$ & $3,1(2,7-3,6)$ & $6,8(<0,001)$ & $7,7(<0,001)$ \\
\hline$>6$ & $10,0(9,5-10,4)$ & $44,0(42,9-45,1)$ & $17,6(16,8-18,5)$ & $25,6(24,6-26,5)$ & $2,8(2,5-3,1)$ & & \\
\hline \multicolumn{8}{|c|}{ Tenencia de seguro de salud } \\
\hline Sí & $7,9(7,5-8,2)$ & $43,9(43,0-44,9)$ & $20,4(19,6-21,1)$ & $24,9(24,0-25,7)$ & $3,0(2,7-3,3)$ & $0,7(0,263)$ & $1,8(0,076)$ \\
\hline No & $7,3(6,5-8,2)$ & $45,4(43,0-47,7)$ & $18,5(16,6-20,4)$ & $26,1(24,0-28,2)$ & $2,7(2,1-3,3)$ & & \\
\hline \multicolumn{8}{|l|}{$\begin{array}{l}\text { Nivel educativo del } \\
\text { responsable }\end{array}$} \\
\hline Sin nivel educativo & $12,7(10,0-15,5)$ & $35,9(31,2-40,6)$ & $8,4(6,19-10,5)$ & $42,4(37,7-47,0)$ & $0,6(0,02-1,2)^{\dagger}$ & $7,0(<0,001)$ & $20,4(<0,001)$ \\
\hline Primaria & $9,7(8,9-10,5)$ & $45,1(43,2-46,9)$ & $11,7(10,5-12,8)$ & $32,1(30,6-33,9)$ & $1,4(1,0-1,8)$ & & \\
\hline Secundaria & $7,8(7,4-8,3)$ & $46,6(45,2-48,0)$ & $19,4(18,3-20,5)$ & $23,8(22,6-25,1)$ & $2,4(2,0-2,7)$ & & \\
\hline Superior & $5,7(5,2-6,3)$ & $40,7(39,2-42,3)$ & $28,8(27,4-30,3)$ & $19,6(18,2-21,0)$ & $5,2(4,6-5,8)$ & & \\
\hline \multicolumn{8}{|c|}{ Índice de riqueza del hogar } \\
\hline Muy pobre & $12,2(11,2-13,1)$ & $39,7(38,0-41,5)$ & $11,7(10,7-12,7)$ & $35,1(33,5-36,7)$ & $1,3(1,0-1,6)$ & $7,2(<0,001)$ & $17,8(<0,001)$ \\
\hline Pobre & $7,4(6,8-8,1)$ & $49,0(47,3-50,8)$ & $18,2(16,9-19,5)$ & $23,1(21,6-24,7)$ & $2,2(1,7-2,7)$ & & \\
\hline Medio & $6,2(5,6-6,9)$ & $47,6(45,6-49,7)$ & $21,7(20,3-23,2)$ & $21,7(20,0-23,3)$ & $2,8(2,2-3,3)$ & & \\
\hline Rico & $5,8(5,1-6,5)$ & $42,1(39,8-44,4)$ & $25,3(23,1-27,5)$ & $22,7(20,7-24,7)$ & $4,1(3,3-4,9)$ & & \\
\hline Muy rico & $5,0(4,1-5,9)$ & $41,6(39,0-44,3)$ & $29,5(27,2-31,9)$ & $18,1(15,9-20,2)$ & $5,8(4,8-6,8)$ & & \\
\hline \multicolumn{8}{|l|}{$\begin{array}{l}\text { Dominio geográfico de } \\
\text { residencia }\end{array}$} \\
\hline Lima Metropolitana & $5,9(5,2-6,6)$ & $41,5(39,3-43,8)$ & $23,7(21,9-25,6)$ & $24,1(22,1-26,2)$ & $4,8(4,0-5,5)$ & $3,5(<0,001)$ & $11,3(<0,001)$ \\
\hline Resto de costa & $6,8(6,3-7,4)$ & $49,9(48,3-51,4)$ & $20,6(19,2-21,9)$ & $20,2(19,0-21,5)$ & $2,5(2,1-2,9)$ & & \\
\hline Sierra & $9,7(9,1-10,4)$ & $37,0(35,5-38,4)$ & $20,5(19,3-21,6)$ & $30,9(29,6-32,3)$ & $1,9(1,6-2,2)$ & & \\
\hline Selva & $9,4(8,3-10,5)$ & $51,6(49,6-53,5)$ & $12,4(11,3-13,5)$ & $24,6(23,0-26,2)$ & $2,0(1,6-2,4)$ & & \\
\hline \multicolumn{8}{|l|}{ Área de residencia } \\
\hline Urbana & $6,4(6,0-6,7)$ & $45,6(44,5-46,8)$ & $22,4(21,5-23,3)$ & $22,1(21,2-23,1)$ & $3,5(3,1-3,8)$ & $5,6(<0,001)$ & $9,1(<0,001)$ \\
\hline Rural & $11,9(11,0-12,8)$ & $39,8(38,1-41,5)$ & $13,3(12,3-14,4)$ & $33,6(32,0-35,1)$ & $1,4(1,1-1,7)$ & & \\
\hline
\end{tabular}

Todas las estimaciones incluyeron el factor de ponderación y características muestrales de la ENDES 2018

Diferencia A: diferencia en categorías extremas de no cepillado

Diferencia B: diferencia en categorías extremas de cantidad inadecuada de flúor en pasta dental

* Obtenida mediante una prueba $\mathrm{t}$

† Estimación con coeficiente de variación mayor a $15 \%$ considerada como referencial

IC95\%: intervalo de confianza al 95\%

de padres con nivel educativo superior $(28,8 \%)$, y con mayor índice de riqueza en el hogar $(29,8 \%)$, posiblemente porque este sector de la población tiene mayor capacidad económica para adquirir pastas dentales para niños, que son más caras y en su mayoría contienen cantidades inadecuadas de flúor ${ }^{(11,12)}$. Asimismo, el dominio geográfico con mayor cantidad de uso inadecuado fue Lima Metropolitana $(23,7 \%)$ y el área urbana $(22,4 \%)$. La razón detrás de esta distribución estaría en el elevado número de pastas dentales con contenido inadecuado de flúor que se expenden en la ciudad de Lima ${ }^{(11,12)}$, situación similar a lo reportado en otras ciudades de la costa peruana ${ }^{(21,22)}$.

En la interpretación de nuestros resultados, se debe considerar que los datos utilizados se obtuvieron mediante entrevistas con posibles sesgos de memoria e información. 
Sin embargo, las encuestadoras determinaron el nivel de flúor mediante observación directa de la pasta dental. Además, en un buen porcentaje de los evaluados no fue posible corroborar la información, pues el participante no mostró el tubo de pasta dental, reportó no utilizarla o la información era ilegible. Pese a las limitaciones, este es uno de los primeros estudios en Perú en explorar el uso de pastas dentales según concentraciones de flúor, con representatividad nacional.

En conclusión, un porcentaje considerable de los niños peruanos no se cepillan los dientes, con mayor frecuencia en menores de cinco años, no atendidos en servicios odontológicos en los seis meses previos a la encuesta, hijos de padres sin nivel educativo, niños en situación de pobreza, residentes de la Sierra y zonas rurales. Además, un gran número de niños de nuestro país sigue empleando pastas dentales con contenidos inadecuados de flúor, observándose gradientes según la edad, uso de servicios odontológicos, nivel educativo del responsable, índice de riqueza en el hogar, dominio geográfico de residencia y área de residencia.

El superar este problema requiere de la instauración de una política multisectorial que actúe a nivel de todos sus actores sociales, partiendo de la actualización de la norma técnica (Resolución Ministerial No 454-2001-SA/DM), y la regulación de su cumplimiento a nivel de los fabricantes, a cargo de la Dirección General de Medicamentos Insumos y Drogas (DIGEMID). Asimismo, es necesario fortalecer la promoción de la recomendación del cepillado dental y uso de pastas dentales con contenido de flúor mayor a 1000 ppm desde el ámbito universitario (formación de profesionales de salud), escolar (niños, niñas y adolescentes) y campañas a nivel del MINSA, dirigidas a la sensibilización a nivel de los usuarios de salud y profesionales de salud.

Agradecimientos: al Instituto Nacional de Estadística e Informática (INEI), por poner las bases de datos de la ENDES 2018 a disposición de los investigadores en su página web (http://iinei. inei.gob.pe/microdatos/).

Contribuciones de autoría: AHV y DA idearon el estudio. AHV procesó los datos y elaboró el código en RStudio. Ambos autores participaron en la interpretación de los resultados, redacción del manuscrito, aprobaron la versión final y son responsables de los contenidos del manuscrito.

Fuentes de financiamiento: autofinanciado.

Conflictos de interés: AHV es editor adjunto de la RPMESP y no tuvo participación en ninguna etapa del proceso editorial posterior al envío del presente artículo, asumiendo únicamente el rol de autor. DA declara no tener conflictos de interés.

\section{REFERENCIAS BIBLIOGRÁFICAS}

1. World Health Organization. Oral health [Internet]. [citado el 2 de octubre de 2019]. Disponible en: https://www.who.int/ news-room/fact-sheets/detail/oral-health

2. Vos T, Abajobir AA, Abate KH, Abbafati C, Abbas KM, Abd-Allah F, et al. Global, regional, and national incidence, prevalence, and years lived with disability for 328 diseases and injuries for 195 countries, 1990-2016: a systematic analysis for the Global Burden of Disease Study 2016. Lancet. 2017;390(10100):1211-59. doi: $10.1016 / 50140-6736(17) 32154-2$.

3. Ministerio de Salud. Prevalencia nacional de caries dental, fluorosis del esmalte y urgencia de tratamiento en escolares de 6 a 8, 10, 12 y 15 años. Perú. 2001-2002 [Internet]. Lima: Dirección General de Epidemiología; 2005 [citado 2 de octubre de 2019]. Disponible en: http://www. dge.gob.pe/publicaciones/pub_caries/ prevalencia_caries.pdf

4. Ortiz-León F. Perfil epidemiológico de salud bucal en niños atendidos en el Seguro Social del Perú. Odontol Pediatr. 2014;13(2):94-103.

5. Benzian $\mathrm{H}$, Williams $\mathrm{D}$. The challenge of oral disease: a call for global action. The oral health atlas 2nd. ed [Internet]. Geneva: FDI World Dental Federation; 2015 [citado el 5 de octubre de 2019]. Disponible en: https://www.fdiworlddental.org/sites/ default/files/media/documents/complete_ oh_atlas.pdf

6. Walsh T, Worthington HV, Glenny A-M, Marinho VC, Jeroncic A. Fluoride toothpastes of different concentrations for preventing dental caries. Cochrane Database Syst Rev. 2019;3:CD007868. doi: 10.1002/14651858.CD007868.pub3.

7. Cury JA, Tenuta LMA. Evidence-based recommendation on toothpaste use. Braz Oral Res. 2014;28 Spec No:1-7. doi: 10.1590/S1806-83242014.50000001.

8. NHS. Fluoride [Internet]. NHS [citado el 2 de octubre de 2019]. Disponible en: https://www.nhs.uk/conditions/fluoride/

9. Ministerio de Salud. Aprueban Norma Técnica Sanitaria para la Adición de Fluoruros en Cremas Dentales, Enjuagatorios y otros productos utilizados en la Higiene Bucal. Ministerio de Salud; 2001. Report No.: Resolución Ministerial N ${ }^{\circ}$ 454-2001SA/DM. Lima: MINSA; 2001 [citado el 5 de octubre de 2019]. Disponible en: http://www.digemid.minsa.gob.pe/ UpLoad/UpLoaded/PDF/RESOLU-
CIONMINISTERIALN454-2001-SA_ DM.pdf

10. Ministerio de Salud. Guía de práctica clínica para la prevención, diagnóstico y tratamiento de la caries dental en niñas y niños [Internet]. Lima: Dirección General de Intervenciones Estratégicas en Salud Pública, Ministerio de Salud; 2017 [citado el 5 de octubre de 2019]. Disponible en: http://bvs.minsa.gob.pe/ local/MINSA/4195.pdf

11. Chávez BA, Vergel GB, Cáceres CP, Perazzo MF, Vieira-Andrade RG, CuryJA. Fluoride content in children's dentifrices marketed in Lima, Peru. Braz Oral Res. 2019;33:e051. doi: 10.1590/18073107bor-2019.vol33.0051.

12. Hinostroza-Ninahuanca JL, SerranoCabana LC, Serrano Cabana RA. Características de las pastas dentales para niños comercializadas en Lima, 2019 [Tesis para obtener título de cirujano dentista]. Lima: Universidad Peruana Cayetano Heredia. [citado el 5 de octubre de 2019]. Disponible en: http:// repositorio.upch.edu.pe/bitstream/ handle/upch/6608/Caracteristicas_ HinostrozaNinahuanca_Jackeline. $\mathrm{pdf}$ ? sequence $=1$ \&isAllowe $\overrightarrow{\mathrm{d}}=\mathrm{y}$ 
13. Oberoi SS, Sharma G, Oberoi A. A cross-sectional survey to assess the effect of socioeconomic status on the oral hygiene habits. J Indian Soc Periodontol. 2016;20(5):531-42. doi: 10.4103/0972$124 X .201629$.

14. Goldman AS, Yee R, Holmgren CJ, Benzian H. Global affordability of fluoride toothpaste. Global Health. 2008;4:7. doi: 10.1186/1744-8603-4-7.

15. Rojas Camacho F. Cepillado dental en niños de 1 a 11 años según la Encuesta Demográfica y Salud Familiar a nivel nacional Perú, 2015 [Tesis para obtener título de cirujano dentista]. León Manco R, editor. Universidad Peruana Cayetano Heredia. [citado el 5 de octubre de 2019]. Disponible en: http://repositorio.upch. edu.pe/bitstream/handle/upch/1010/ Cepillado_RojasCamacho_Fatima. pdf ?sequence $=5 \&$ is Allowed $=y$

16. Agostini BA, Machry RV, Teixeira CR da S, Piovesan C, Oliveira MDM, Bresolin CR, et al. Self-perceived oral health influences tooth brushing in preschool children. Braz Dent J. 2014;25(3):248-52.

17. Shaghaghian $S$, Zeraatkar M. Factors Affecting Oral Hygiene and Tooth
Brushing in Preschool Children, Shiraz/ Iran. J Dent Biomater. 2017;4(2):394-402.

18. Marshman Z, Ahern SM, McEachan RRC, Rogers HJ, Gray-Burrows KA, Day PF. Parents' Experiences of Toothbrushing with Children: A Qualitative Study. JDR Clin Trans Res. 2016;1(2):122-30. doi: $10.1177 / 2380084416647727$.

19. Vallejos-Sánchez AA, Medina-Solís CE, Maupomé G, Casanova-Rosado JF, Minaya-Sánchez M, VillalobosRodelo JJ, et al. Sociobehavioral factors influencing toothbrushing frequency among schoolchildren. J Am Dent Assoc. 2008;139(6):743-9. doi: 10.14219/jada. archive.2008.0256.

20. Thornton-Evans G, Junger ML, Lin M, Wei L, Espinoza L, Beltran-Aguilar E. Use of Toothpaste and Toothbrushing Patterns Among Children and Adolescents-United States, 2013-2016. MMWR Morb Mortal Wkly Rep. 2019;68(4):87-90. doi: 10.15585/mmwr.mm6804a3.

21. Salvatierra García SD. Evaluación de la concentración de fluoruro contenido en los dentífricos bucales fluorados para niños, comercializados en el distrito de Trujillo, 2013 [Tesis para optar el grado de bachiller en Estomatologia]. Lima: Universidad Nacional de Trujillo; 2014 [citado el 6 de octubre de 2019]. Disponible en: http:// dspace.unitru.edu.pe/bitstream/handle/ UNITRU/612/SalvatierraGarcia_S. pdf ?sequence $=1$ \&isAllowed $=y$

22. Atanacio Vara NR. Concentración de flúor de dentífricos comercializados en la ciudad de Chimbote-2018 [Tesis para optar el titulo de cirujano dentista]. Trujillo: Universidad Catolica Los Angeles Chimbote; 2019 [citado el 6 de octubre de 2019]. Disponible en: http://repositorio.uladech.edu.pe/ handle/123456789/10214

Correspondencia: Akram Abdul Hernández Vásquez

Dirección: Universidad San Ignacio de Loyola, Av. La Fontana 550, La Molina, Lima, Perú. Teléfono: (511) 3171000

Correoelectrónico:ahernandez@usil.edu.pe

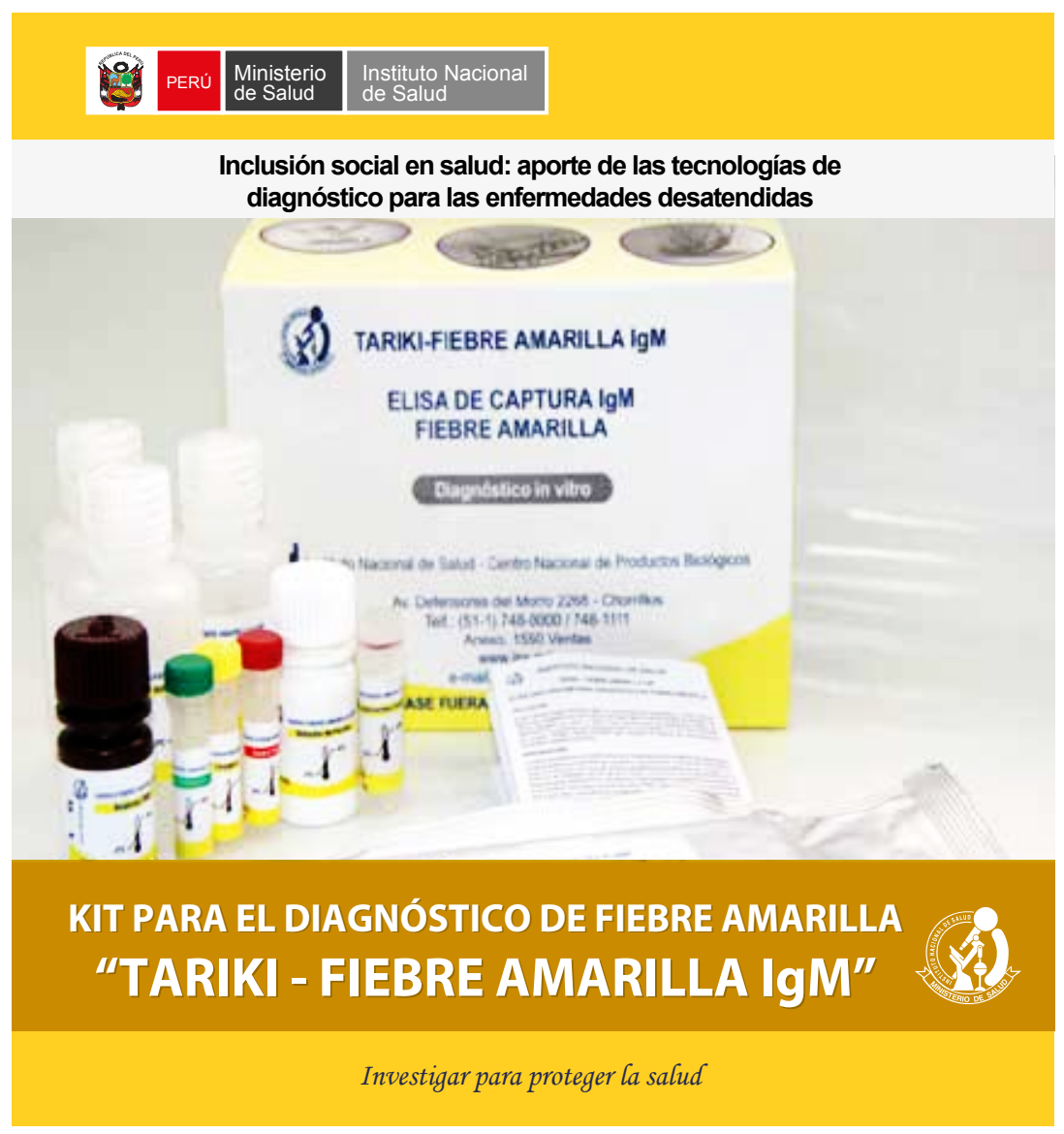

